\title{
TeachMeet: Librarians learning from each other
}

Niamh Tumelty, Isla Kuhn and Katie Birkwood

In summer 2010, Isla Kuhn, Reader Services Librarian at Cambridge University Medical Library, blogged about a particular type of event popular among school teachers, and wondered whether this 'TeachMeet' idea would be of interest to librarians (Kuhn, 2010). Several other librarians at the University of Cambridge responded enthusiastically and LibTeachMeet was born.

\section{So what is TeachMeet?}

First established in 2005 (Hallahan, 2010), TeachMeet is an "unconference" organised by teachers, for teachers. These open, friendly and loosely-structured events provide an opportunity for teachers to share classroom experiences and to learn from each other. The idea is that everyone is an active participant in the event - even those not presenting are "enthusiastic lurkers" as opposed to passive "attendees".

TeachMeets are free to attend, but sponsorship may be sought to cover costs such as venue hire and provision of refreshments. The events themselves consist of 7-minute micro presentations and 2-minute nano presentations. Presenters are encouraged to talk about a tool or technique they have tried themselves. Presentation topics are usually registered in advance using a wiki, but the order of speakers is selected at random on the day. There are no key note speakers or sales pitches and participants are welcome to come and go throughout the presentations. Presentations can be in any style or format but the original TeachMeets encouraged the use of more imaginative presentation techniques than powerpoint presentations. 


\section{Why TeachMeet for Librarians?}

Recent research undertaken on behalf of the Chartered Institute of Library and Information Professionals (Research by Design Ltd., 2010) found that $71 \%$ of information professionals use training skills and $50 \%$ use teaching skills in their current roles, while $63 \%$ expect to be using these skills a lot or a little more in ten years' time. Despite the evident importance of the educational role of the librarian, educational theory and teaching skills are not included as a compulsory component of many librarianship courses in the UK, so information professionals require alternative opportunities to develop these skills. Just as many teachers work on their own in their classrooms, many librarians teach in isolation with little opportunity to share advice and experiences. Alternative methods of training and sharing are needed and this is where the TeachMeet model can help.

TeachMeets are quick and cheap to organise, given the emphasis on informality, and offer a convenient opportunity to meet up with colleagues outside expensive conferences or other large-scale training events. The welcoming and accessible format of these events facilitates the sharing of experiences without intimidating the first-time presenter.

\section{The First LibTeachMeet}

A number of factors combined to make the first Cambridge LibTeachMeet a success. As part of a 23 Things programme which took place in the summer of 2010 nearly 100 librarians across the University of Cambridge were blogging about a set of social media tools (23 Things Cambridge, 2010).

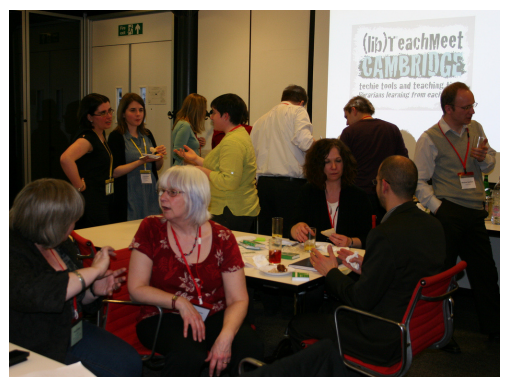

Networking at the second Cambridge LibTeachMeet. Photo with permission of Norman Minter (photographer) and Schlumberger Research Centre. 
staff of the many different libraries of the University of Cambridge. It is therefore not surprising that when Isla Kuhn blogged about TeachMeet, a 5-person organising committee formed itself in the comments of the blog post. It is now hard to imagine that only two members of this committee had met in person before the first TeachMeet planning meeting! As the 23 Things programme drew to a close, participants began to ask how this new level of communication could be continued. TeachMeet became something to look forward to, an opportunity to continue to develop connections built during 23 Things as well as a chance to share best practice and learn from colleagues in neighbouring libraries.

The first planning meeting took place in August 2010. A date and time were picked, and a page was created on the TeachMeet wiki (Cambridge Librarian TeachMeet, 2010.) Each member of the organising committee blogged about the event, and the hashtag \#camlibtm was used on Twitter to start raising awareness of it. By the time the 23 Things closing ceremony came around, the TeachMeet team was ready to announce the date of the first Cambridge LibTeachMeet. Bookings opened in August 2010 and began to fill even before a venue had been announced. This free event was fully booked well in advance and a selforganised waiting list formed on the wiki. There was no difficulty in finding sufficient speakers, and in fact there was just enough time to accommodate all of the volunteers. 


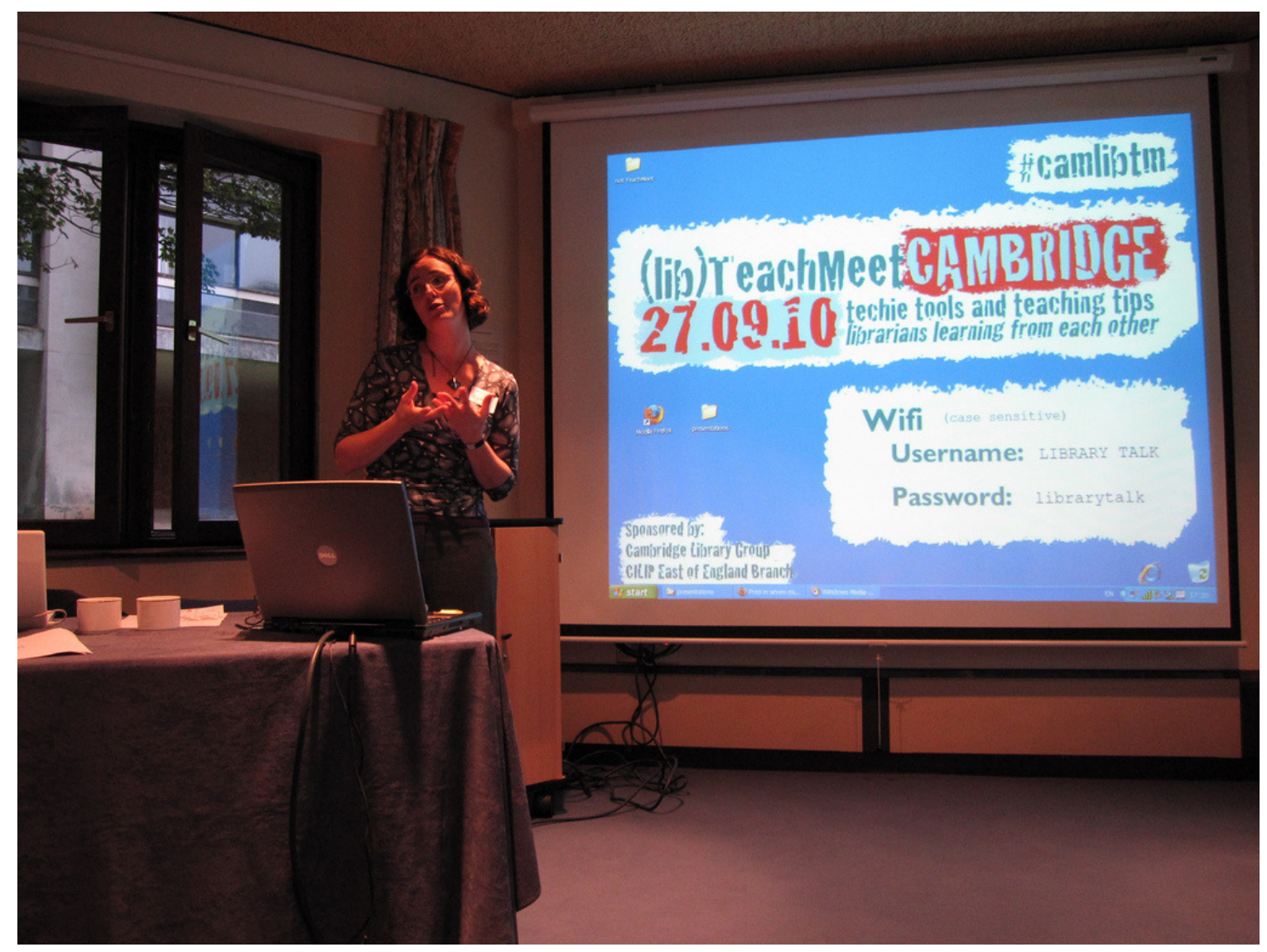

Celine Carty chairs the first Cambridge LibTeachMeet. Photo courtesy of the Cambridge TeachMeet Team

The first LibTeachMeet took place in St John's College, Cambridge on Monday 27th September 2010. Refreshments were provided thanks to sponsorship by the Cambridge Library Group and CILIP East of England. 50 people signed up to attend (though attendance on the day was 40 ). There were 11 presentations covering the following topics:

) Library inductions using the Cephalonian method

> Some informal Facebook research

> 'Getting them early': database training in inductions

Prezi: an improvement on Powerpoint?

> From School to Uni: transition skills

> Adobe Captivate video tutorials

> The new LAT (Librarians as Teachers) network

> 'Learning Hub' (English Faculty Library's blog for online help and tutorials) 
> Using Flashmeeting videoconference technology

$>$ Setting up a project blog

Solving three banner problems.

All the presentations were filmed and made available via YouTube (http://www.youtube.com/camlibtmvideo). Powerpoint slides were posted to SlideShare (http://www.slideshare.net/camlibtm), photos of the event were added to Flickr (http://www.flickr.com/photos/camlibtm) and blog posts and other relevant web pages were tagged in Delicious (http://www.delicious.com/search?p=camlibtm\&chk=\&context=all|| $\left.\& f r=d e l \_i c i o \_u s \& \mid c=1\right)$.

\section{Response to the first LibTeachMeet}

Participants were invited to give their general impressions of the event end of the evening, and the response was overwhelmingly positive: $24 \%$ of attendees enjoyed the event "hugely" and 73\% thought it was good fun (Cambridge Librarians' TeachMeet, 2010). Attendees were also asked which of the ideas presented they thought were most likely to be of use to them.

A qualitative survey two weeks later provided an opportunity for more reflective feedback and received an excellent response rate (87\%). Participants were asked about their expectations prior to the event and whether those expectations were met, and the responses received were well-considered and extremely constructive.

When asked if they had made use of at least one of the ideas presented 28 of 35 respondents said yes, and many had explored more than one. For those who had not explored any of the ideas the consistent explanation was "lack of time". 
When asked if they would consider speaking at the next event, 29/35 respondents said yes, often citing the relaxed atmosphere as a good way for newcomers to get experience of public speaking. However most respondents seemed doubtful they had anything of interest to share: a typical response was "Yes, if I had something to speak about".

Table 1: Examples of feedback regarding expectations of the first Cambridge LibTeachMeet

\begin{tabular}{|c|c|}
\hline $\begin{array}{l}\text { What were your } \\
\text { expectations? }\end{array}$ & $\begin{array}{l}\text { Do you feel these expectations were met, and if } \\
\text { so, why? }\end{array}$ \\
\hline A few teaching tips & $\begin{array}{l}\text { Actually, got more out of it than that. The opportunities } \\
\text { to chat with others were good. }\end{array}$ \\
\hline $\begin{array}{l}\text { I thought it might be a bit } \\
\text { "lecturey" and to do with } \\
\text { teaching techniques. }\end{array}$ & $\begin{array}{l}\text { No, and I was pleased they weren't. I enjoyed it and } \\
\text { found it much more interesting than I thought I would. } \\
\text { I liked the variety of content and presentation style, } \\
\text { and the brevity of each presentation. }\end{array}$ \\
\hline $\begin{array}{l}\text { I'd been to a similar thing } \\
\text { before - but more tech \& } \\
\text { design orientated and } \\
\text { private sector - and from } \\
\text { that my expectations were } \\
\text { really quite low. }\end{array}$ & $\begin{array}{l}\text { No, they were exceeded. I thought the length of time } \\
\text { slots were very good. I felt it helped the more nervous } \\
\text { presenters to know that this would literally be over in } \\
\text { a matter of minutes, literally, which in turn seemed to } \\
\text { relax them. The content was a good mixture and there } \\
\text { was plenty of value to be had, even if someone was } \\
\text { presenting something I was familiar with, I could } \\
\text { either learn from a different perspective or digest } \\
\text { some of what had gone before. } \\
\text { And also, there's nothing wrong with networking and } \\
\text { building on the budding relationships formed during } \\
\text { Cam23. }\end{array}$ \\
\hline
\end{tabular}




\section{The Second Cambridge LibTeachMeet}

The organising team gained four more enthusiastic volunteers and planning for the second

Cambridge LibTeachMeet soon began. This time, a new website was established for

Cambridge LibTeachMeets, and bookings were handled using Eventbrite. Feedback from participants at the first event was taken into account, and the second event took place on from 6-8pm on 29th March 2011 in Schlumberger Research Centre, Cambridge. This venue was slightly bigger, allowing for 60 participants and café-style seating arrangements.

Sponsorship for the second event was provided by Schlumberger Research Centre and the Arcadia Project at Cambridge University Library.

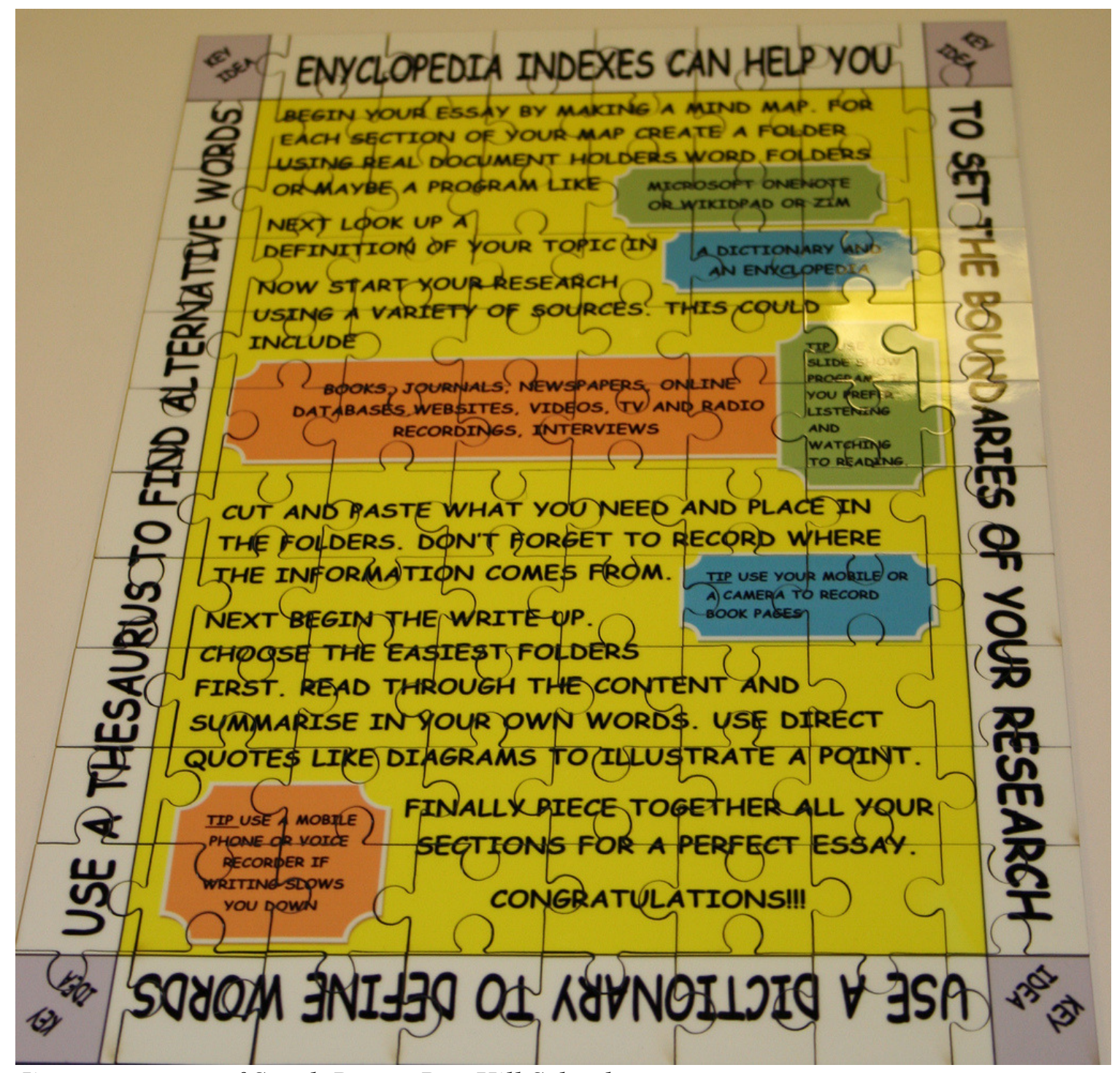

Jigsaw courtesy of Sarah Pavey, Box Hill School. 
Presentation timings were more flexible and more opportunities for networking were built into the event. The conscious effort to widen participation beyond the University of Cambridge paid off, with attendees coming from school, university, medical, public, and corporate libraries and information services. The wider range of participants resulted in a different balance of topics, from QR codes in medical libraries and the use of special collections in teaching to jigsaws as a tool for developing essay writing skills and "Debunking the myth of the Google generation". Again, the event was publicised and recorded using a range of social media services, and this time the event was full within hours of opening booking.

The evening began with an icebreaker, "Human Bingo", to encourage conversation with librarians from different sectors. Each attendee had to fill a bingo sheet with signatures of people that worked in different types of libraries, from different countries and with different hobbies. An opportunity was provided for round table sessions half-way through the presentations, to allow for discussion of topics relating to the issues raised over refreshments, but these were not necessary as the participants had by this stage found enough to talk about without additional encouragement!

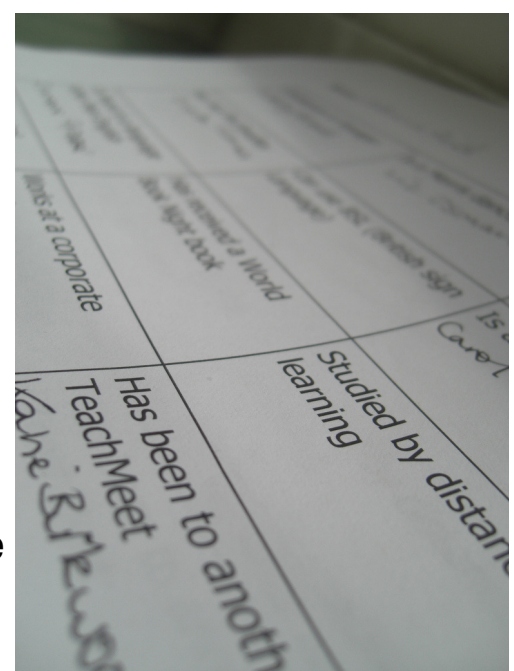

Human Bingo. Photo courtesy of Norman Minter.

Feedback received suggests that a much better balance was achieved between presentations and networking at this second event.

\section{LibTeachMeet Continues}

Because the Cambridge events were publicised and documented using social media, news quickly spread of this new teaching event. A post on the CILIP Update blog further facilitated the dissemination of this 'TeachMeet' idea (Tumelty

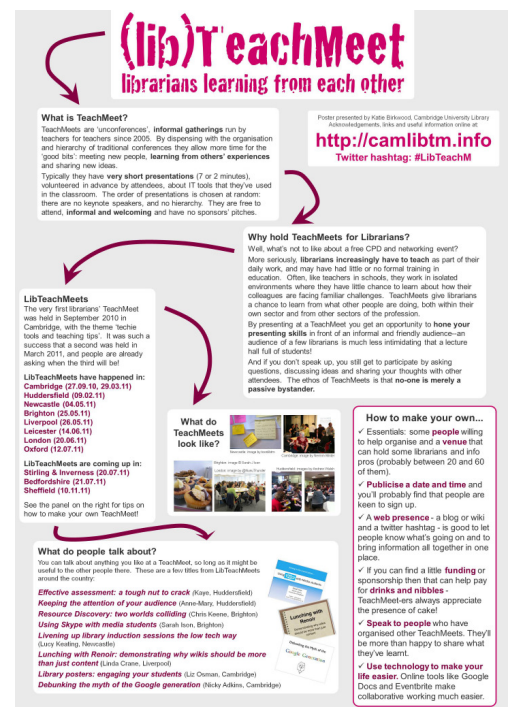


2011). Even before the second Cambridge Librarian TeachMeet had taken place, University of Huddersfield librarians held their own event. LibTeachMeets have now taken place throughout the UK, from Newcastle to Brighton and Liverpool to London. Papers were presented at LILAC (Tumelty and Birkwood, 2011) and EAHIL (Kuhn, 2011b) conferences, and are helping to spread the idea beyond the UK. A TeachMeet has taken place in Limerick, Ireland, and interest has been expressed in organising similar events in Sweden and Austria. Presentations were also given more locally in Oxford (Osman, 2011) and Cambridge (Kuhn, 2011a) and a poster session was presented at CILIP's Umbrella conference (Birkwood, 2011). In most cases the impetus has come from academic librarians, but the museum and archives sector have adopted the idea too: TeachMeet Museums took place in February 2011. A public google calendar has been created listing all the TeachMeets planned. While each TeachMeet will likely have its own twitter hashtag, at the time of writing, tweets talking about TeachMeets in general can use \#LibTeachM.

\section{Conclusions}

TeachMeets are an effective and inexpensive way to enable sharing of experience and networking of colleagues locally across the various sectors. They support continuing professional development for the organisers as well as the attendees, and can be a supportive environment to encourage first time presenters.

The genesis, development and dissemination of the LibTeachMeet idea all happened via social media. Social media tools, like blogs, Twitter, wikis, Google Docs and Eventbrite, have made the organisation of informal networking and training events much simpler and enabled the concept to spread further and more rapidly. The success of LibTeachMeets ultimately demonstrates that a real power of social media is in enabling new and better forms of off-line interaction and participation, in addition to the creation of online networks. 


\section{Useful websites}

www.teachmeet.org.uk

www.camlibtm.info

\section{Bibliography}

23 Things Cambridge, 2010. The Cambridge 23 Things. 23 Things Cambridge. Available at: http://23thingscambridge.blogspot.com/p/list-of-23-things.html [Accessed July 13, 2011].

Birkwood, K., 2011. (lib)TeachMeet: librarians learning from each other. Available at: http://www.camlibtm.info/2011/07/12/libteachmeet-at-umbrella/ [Accessed July 13, 2011]. Cambridge Librarian TeachMeet, 2010. Cambridge Librarian TeachMeet. TeachMeet teachers sharing ideas with teachers. Available at: http://teachmeet.pbworks.com/w/page/28149182/Cambridge-Librarian-TeachMeet [Accessed July 13, 2011].

Cambridge Librarians' TeachMeet, 2010. Quick evaluation of the event. Available at: http://www.slideshare.net/camlibtm/quick-evaluation-of-the-event [Accessed July 13, 2011].

Hallahan, I., 2010. TeachMeet - The Story So Far.... The H-Blog. Available at: http://hblog.me.uk/?p=161 [Accessed July 13, 2011].

Kuhn, I., 2011a. Cambridge Librarians Teachmeet. Available at: http://prezi.com/e9wuhvjahw4/cambridge-librarians-teachmeet/ [Accessed July 13, 2011]. Kuhn, I., 2010. teach me(et)! musings of a medical librarian. Available at: http://ilk21.wordpress.com/2010/07/03/teach-meet/ [Accessed July 13, 2011]. Kuhn, I., 2011b. TeachMeet - Librarians learning from each other. Available at: http://prezi.com/a7jk_zr5j6to/eahil-teachmeet/[Accessed July 13, 2011]. Osman, L., 2011. CamlibTM. Available at: http://prezi.com/pxbcuon6g428/camlibtm/ 
[Accessed July 13, 2011].

Research by Design Ltd., 2010. Defining our professional future: Report to CILIP Council, Birmingham. Available at: http://www.cilip.org.uk/getinvolved/cilipfuture/Pages/default.aspx [Accessed July 13, 2011]. TeachMeet.org.uk, Teachmeet: Teachers sharing ideas with teachers. Available at: http://www.teachmeet.org.uk/ [Accessed July 13, 2011].

Tumelty, N., 2011. How to run your own LibTeachMeet. CILIP Update. Available at: http://communities.cilip.org.uk/blogs/update/archive/2011/03/17/how-to-run-your-ownlibteachmeet-160-by-niamh-tumelty.aspx [Accessed July 13, 2011]. Tumelty, N. \& Birkwood, K., 2011. TeachMeet - librarians learning from each other. Available at: http://www.slideshare.net/camlibtm/teachmeet-librarians-learning-from-eachother-niamh-tumelty-and-katie-birkwood [Accessed July 13, 2011]. 